\title{
Child and Adolescent Psychiatry and Mental Health reviewer acknowledgement 2014
}

Jörg M Fegert

\section{Contributing reviewers}

The editors of Child and Adolescent Psychiatry and Mental Health would like to thank all of our reviewers who have contributed to the journal in volume 8 (2014).

Marc Allroggen

Germany

Martijn Arns

Netherlands

Josephine Barbaro

Australia

Claus Barkmann

Greenland

Sheri Bauman

United States of America

Michele Baumann

Luxembourg

Myron Belfer

United States of America

Vashti Berry

United Kingdom

Maria Teresa Botello-Harbaum

United States of America

David Buchbinder

United States of America

Larry Burd

United States of America

Marcy Burstein

United States of America

Franco Cavallo

Italy

\author{
David Chambers \\ United States of America \\ Yiong Huak Chan \\ Singapore \\ Deena Chisolm \\ United States of America \\ Joanna Ting Wai Chu \\ New Zealand
}

Antonio Clavenna

Italy

Paula Cloutier

Canada

Melissa Cortina

United Kingdom

Georgina Cox

Australia

Michelle Dawson

Canada

Rafael de Assis da Silva

Brazil

Arden Dingle

United States of America

Joseph Durlak

United States of America

Pia Enebrink

Sweden
Elif Ercan
Turkey

Philip Fisher

United States of America

Carol Fitzpatrick

Ireland

Bacy Fleitlich-Bilyk

Brazil

Lila Fleming

United States of America

Marjolein Fokkema

Netherlands

Daniel Fung

Singapore

Holger Gevensleben

Germany

Laurence Greenhill

United States of America

Johannes Haerting

Germany

Charlotte Hanisch

Germany

Lisa Harryson

Sweden

Nina Heinrichs

Germany

Correspondence: capmh.editorial@uniklinik-ulm.de

Department of Child and Adolescent Psychiatry and Psychotherapy,

University of UIm, Steinhoevelstr. 5, Ulm 89075, Germany 
Pieter J Hoekstra

Netherlands

Embry Howell

United States of America

Tina In-Albon

Germany

Sigrid James

United States of America

Bruce Jonas

United States of America

Jennifer Jordan

New Zealand

Niina Junttila

Finland

Jon Jureidini

Australia

Espérance Kashala

Congo Democratic Republic

Ferdinand Keller

Germany

David Kemp

United States of America

Bonnie Kerker

United States of America

Suzanne Kerns

United States of America

Praveen Khairkar

India

Christian Kieling

Brazil

Hyun-Sil Kim

South Korea

Bryan King

United States of America

Michael Kohn

Australia

Anne Katrin Künster

Germany

Natasha Latzman

United States of America

Elizabeth Letourneau

United States of America

Ching-Hua Lin

Taiwan
Yu-Ju Lin

Taiwan

Arnold Lohaus

Germany

Astri J. Lundervold

Norway

Aristides Machado-Rodrigues

Portugal

Stefania Maggi

Canada

Rajneesh Mahajan

United States of America

Andres Martin

United States of America

Therese Mathews

United States of America

Jon McClellan

United States of America

Christopher McDougle

United States of America

Joe McNamara

United States of America

Anilena Mejia

United Kingdom

Susannah Melville

United Kingdom

Eugen Mengel

Germany

Inmaculada Moreno García

Spain

Tally Moses

United States of America

Stella Muthuri

Canada

Kerstin Neander

Sweden

Dennis Nitkowski

Germany

Ingrid Landgraff Oestlie

Norway

Thomas Olino

United States of America

Susanna Payne

United Kingdom
Geraldine Pearson

United States of America

Wendy Plante

United States of America

Paul L. Plener

Germany

Guilherme Polanczyk

Brazil

Maria Cristina Porfirio

Italy

Irina Elizabeth Poslawsky

Netherlands

Jonathan Prince

United States of America

Daniel Radeloff

Germany

Nicola Reavley

Australia

Ruth Reed

United Kingdom

Nicole Reilly

Australia

Matthias Reitzle

Germany

Thiago Rocha

Brazil

Paul Rohde

United States of America

Jeanne Ryan

United States of America

Per-Anders Rydelius

Sweden

Christina Sakai

United States of America

Giovanni Salum

Brazil

Masafumi Sanefuji

Japan

Ursula Sansom-Daly

Australia

Caferi Tayyar Sasmaz

Turkey

Susan Sawyer

Australia 
Erin Schoenfelder

United States of America

Thomas Schofield

United States of America

Fiona Schulte

Canada

Donald Sharpe

Canada

Jordan Sibeoni

France

Inga Dora Sigfusdottir

Iceland

Ilina Singh

United Kingdom

Petros Skapinakis

Greece

Tristram Smith

United States of America

Gottfried Spangler

Germany
Anthony Spirito

United States of America

Fabio Sticca

Switzerland

Lisanne Stone

Netherlands

Carla Stover

United States of America

James Swanson

United States of America

Katalin Szakszon

Hungary

Toshinobu Takeda

Japan

Sarah Tarbox

United States of America

Cristiano Tschiedel Belem da Silva

Brazil

Aina Basilier Vaage

Norway
Mitch van Geel

Netherlands

Benedetto Vitiello

United States of America

Jan Wallander

United States of America

James Waxmonsky

United States of America

Carl Weems

United States of America

Yifeng Wei

Canada

Gunda Wößner

Germany

Peter Zimmermann

Germany 